\title{
Structural analysis of human intestinal alpha-glucosydases: Sucrase-isomaltase and maltase-glucoamylase.
}

\author{
N Nava ${ }^{1}$, D Rose ${ }^{2}$ \\ ${ }^{1}$ University of Waterloo, Waterloo, ${ }^{2}$ Dept of Biology, Univ of Waterloo \\ nenavaro@uwaterloo.ca
}

Sucrase-isomaltase (SI) and maltase-glucoamylase (MGAM) both belong to the glycoside hydrolase family 31 (GH31). These two enzymes each contain two catalytic subunits at the N- and C-termini. Each of the subunits possesses the characteristic $(\beta / \alpha) 8$ barrel catalytic pocket inherent to this family, and all the subunits of these enzymes employ the double displacement mechanism and conserve the two characteristic consensus motifs containing the catalytic residues; the aspartic acid in WIDMNE acts as the catalytic nucleophile while the aspartic acid within the motif HWLGDN functions as the proton donor. While SI and MGAM show high sequence similarity, each subunit of the two enzymes hydrolyzes specific substrates with a level of overlapping activity, presumably to enhance the efficiency of carbohydrate digestion. This suggests that residues outside the catalytic pocket contribute to the substrate affinity. By comparing the structures of the different subunits, the goal of this project is to identify key residues involved in substrate affinity among the four subunits. Initial computational modelling and docking based on structures in hand will be supplemented by new crystallographic or Cryo-EM models of the human enzymes, expressed in yeast cells..

Acta Cryst. (2020). A76, a14 